\title{
A comparative study evaluating C-reactive protein, sputum eosinophils and forced expiratory volume in one second in obese and nonobese asthmatics
}

\begin{abstract}
Introduction: Asthma and obesity are considered inflammatory disorders. Inflammatory markers - sputum eosinophils, C-reactive protein (CRP) and the forced expiratory volume in one second $\left(\mathrm{FEV}_{1}\right)$ were analysed to find their association in obese asthmatics and compared with their asthma control test (ACT) to understand these parameters in this phenotype.

Material and methods: After completing the asthma control test (ACT), the CRP, FEV ${ }_{1}$ and sputum eosinophils of sixty asthmatics were compared to find the association of them in obese and nonobese asthmatics and contrasted with their ACT. The data were analysed using IBM SPSS V20.0, Mann-Whitney U test (non-parametric test), Pearson's correlation coefficient and Fisher's exact test.

Results: We found significant differences for CRP $(P=0.001)$ and sputum eosinophils $(P=0.001)$ between obese and nonobese asthmatics, both higher in obese asthmatics and with a significant association with body mass index (BMI) $(\mathrm{P}<0.05)$. The $\mathrm{FEV}$, levels were independent of the BMI levels of asthmatics. There was a significant correlation between the CRP and sputum eosinophils $(0.52, P=0.001)$ for all asthmatics. There was no significant correlation between $\mathrm{FEV}_{1}$ and sputum eosinophils (nonobese $P=0.120$, obese $P=0.388$ ) and between $F E V_{1}$ and CRP (obese $P=0.423$, nonobese $P=0.358$ ) in both obese and nonobese asthmatics. Obesity had an association $(P=0.001)$ with ACT scores $(\leq 19)$.

Conclusions: Sputum eosinophils and CRP were raised in obese asthmatics and had a positive association with BMI. Obese asthmatics had a poorer subjective asthma control than nonobese asthmatics despite FEV ${ }_{1}$ being independent of the BMI levels. Measuring the systemic inflammatory markers could help in additional interventions in reducing systemic inflammation and thus possibly facilitating better symptom control.
\end{abstract}

Key words: C-reactive protein, sputum eosinophils, $\mathrm{FEV}_{1}$, obese asthmatics, systemic inflammation

Adv Respir Med. 2020; 88: 394-399

\section{Introduction}

Asthma is a syndrome characterised by recurrent episodic airway obstruction, airway inflammation and bronchial hyper-responsiveness. It is a syndrome with a variety of phenotypes, where various precipitating factors result in clinical, physiological and pathological manifestations. The main pathogenesis of asthma is the infiltration of inflammatory cells such as eosinophils, basophils, and CD4 + lymphocytes in the airways [1, 2].

Obesity is also considered an inflammatory disorder conveyed by various systemic inflam- matory mediators like C-reactive protein (CRP) that leads to an increase in circulating levels of the pro-inflammatory cytokines. CRP is also raised in various systemic inflammations such as diabetes, cardiovascular diseases, collagen vascular diseases, malignancies, and also obesity [3-5]. Various studies have shown that severe asthma is more prevalent in obese patients as compared with patients with normal body mass index (BMI) and that BMI is positively associated with asthma severity [6]. Also, BMI correlates positively with the level of asthma control, with more severe asthmatics having a higher BMI than

Address for correspondence: Harish Mahender, Department of Respiratory Medicine, Sree Balaji Medical College and Hospital, Bharath University, Chromepet, Chennai, India;

e-mail: drmharish@gmail.com

DOI: 10.5603/ARM.a2020.0155

Received: 19.04.2020

Copyright (C) 2020 PTChP

ISSN 2451-4934 
those with milder asthma $[7,8]$. Thus, obesity seems to be related with asthma severity, but the mechanisms responsible for this relationship are not yet clarified.

There is growing evidence that asthma and obesity are strongly associated with each other and can alter each other's status [9]. Also, the inflammatory cells, sputum eosinophils and interleukin-5 play a major role in airway inflammation in asthmatics, more so in obese asthmatics who are already in a pro-inflammatory state [10]. Evidence of neutrophilic inflammation of the airways in obese asthmatics has also been documented [11], but the association between the non-neutrophilic airway inflammation in obesity, the systemic inflammation and the lung function remains poorly understood, especially in different phenotypes and endotypes of asthma. As the treatment of asthma has evolved from the airway obstruction-based approach to a tailored-endotype and phenotype-based approach, especially in special subsets of asthma like the obese phenotypes, it helps to know the relationship between the inflammatory markers both systemic (CRP) and airway (sputum eosinophils), with the forced expiratory volume in one second $\left(\mathrm{FEV}_{1}\right)$ in this regard.

As our understanding of various subtypes of asthma has evolved over years, here we try to evaluate the obese asthmatic subtype and the inflammatory markers associated with both the airway (sputum eosinophils) and systemic marker (CRP), and correlate them with their lung function $\left(\mathrm{FEV}_{1}\right)$ and compare the same with nonobese asthmatics. This might help us to understand the systemic inflammatory component of asthma, specifically concerning the obese asthmatic phenotype and the markers associated with it. Correlating these inflammatory markers with the patient's subjective control of symptoms through the asthma control test (ACT) could give us a better understanding of the roles these inflammatory markers play in the symptom control.

The purpose of this cross-sectional study was to evaluate the systemic inflammation using CRP, airway inflammation by measuring sputum eosinophils and the measure of lung function by $\mathrm{FEV}_{1}$ in obese and nonobese asthmatics, compare and correlate these parameters between the obese and nonobese groups. We also try to ascertain whether there is a significant association between the inflammatory markers (sputum eosinophils, CRP) amongst each other and with $\mathrm{FEV}_{1}$ and to compare BMI with the asthma control test (ACT) scores.

\section{Materials and methods}

Sixty asthmatic patients who presented to the outpatient department of Respiratory Medicine, in Sree Balaji Medical College and Hospital, Chennai. were recruited for the study from 2018 to 2019. We aimed to compare and evaluate CRP, FEV ${ }_{1}$ and sputum eosinophils in obese and nonobese asthmatics and to find the association of CRP, $\mathrm{FEV}_{1}$, and sputum eosinophils among each other and compare BMI with the asthma control test scores (ACT) [12].

Patients with a primary diagnosis of asthma according to the Global Initiative for Asthma (GINA) guidelines, 18 years of age or older were included in this study. Individuals with nonreversible airway obstruction on spirometry $(<12 \%$ change in $\mathrm{FEV}_{1}$ ) who were unable to do spirometry due to a history of myocardial infarction, congestive heart failure, coronary artery disease, who had a history of smoking, with known comorbidities like diabetes, cardiovascular diseases, collagen vascular diseases and malignancies were excluded from the study.

All the patients who satisfied the inclusion criteria, filled up the asthma control test and were later told to do pulmonary function test - spirometry, serum CRP and sputum sample after deep coughing in a sterile container. Then sputum quality was assessed using both macroscopic and microscopic criteria [13]. Sputum was stained with eosin and haematoxylin and analysed using microscopy to determine the count of eosinophils expressed in percentage, and counts $\geq 3 \%$ was considered to be high [14], BMI $\geq 30 \mathrm{~kg} / \mathrm{m}^{2}$ were considered obese, and CRP $>1 \mathrm{mg} / \mathrm{dL}$ was considered high-risk. The ACT is a patient-completed questionnaire for individuals above 12 years of age and consists of five items evaluating the preceding 4 weeks (limitation of activities, shortness of breath, awakenings at night, use of reliever medication and patient's perception of asthma control). Each question has five response options, resulting in scores of 1-5. The sum of all scores yields the total ACT score, a score of less than equal to 19 indicates poorly controlled asthma, and a score greater than equal to 20 indicates good asthma control, the maximum being 25 . All the participants had forced expiratory volume 1 second/functional vital capacity $\left(\mathrm{FEV}_{1} / \mathrm{FVC}\right)$ $<70 \%$ with post-bronchodilator reversibility $\mathrm{FEV}_{1}>12 \%$ on spirometry.

Statistical analysis: A total of 60 patients were included in the study. The data were analysed using IBM SPSS V 20.0. Mann-Whitney 
U test (non-parametric test) was performed for comparing outcome variables between obese and nonobese groups, Pearson's correlation coefficient was computed to measure the association of the outcome variables amongst each other and Fisher's exact test was used to find the association of BMI with the asthma control test score. P-value $<0.05$ was considered to be statistically significant.

\section{Results}

There were a total of 60 subjects. Among them, the number of males were 22 (37\%) and females were 38 (63\%). The mean age was 41.48 (SD $=10.98)$. All of the participants were classified as obese $(\mathrm{BMI} \geq 30)$ and nonobese $(\mathrm{BMI}<30)$ (Table 1). Among the 60 subjects, 32 were obese and 28 were nonobese (Table 1).

We found statistically significant differences in CRP $(\mathrm{P}=0.001)$ and sputum eosinophils $(\mathrm{P}=$ $0.001)$ between obese and nonobese asthmatics (Table 1). We noted that the median CRP was higher, $2 \mathrm{mg} / \mathrm{dL}$ for obese asthmatics compared to $1 \mathrm{mg} / \mathrm{dl}$ for nonobese asthmatics (Table 1, Figure 1). Also, the same trend was reflected in median sputum eosinophils, being $6 \%$ for obese asthmatics and $2 \%$ for nonobese asthmatics (Table 1, Figure 2). However, we did not find any significant difference in $\mathrm{FEV}_{1}$ between obese and nonobese asthmatics $(\mathrm{P}=0.882)$ (Table 1$)$.

Among the associations between the parameters themselves (Table 2), we observed that there was a significant correlation between the CRP and sputum eosinophils (0.52, $\mathrm{P}=0.001)$ for all asthmatics (Figure 3). We found no significant correlation between $\mathrm{FEV}_{1}$ and sputum eosinophils (nonobese $\mathrm{P}=0.120$, obese $\mathrm{P}=0.388$ ) and also between $\mathrm{FEV}_{1}$ and CRP (obese $\mathrm{P}=0.423$, nonobese $\mathrm{P}=0.358$ ) in both obese and nonobese asthmatics. Comparing the BMI with the asthma control test scores (Table 3), we noticed that asthma was not controlled (ACT scores $\leq 19$ ) for $94 \%$ of obese asthmatics as compared to only $25 \%$ for nonobese asthmatics, and there was a significant association $(\mathrm{P}=0.001)$ between obesity (BMI) and ACT scores.

Table 1. Comparison of outcome variables between obese and nonobese asthmatics. Values presented as median with interquartile range in parenthesis. * Mann-Whitney-u non-parametric test; $P$ value $<0.05$ - statistically significant

\begin{tabular}{lccc}
\hline Outcome & Non-obese (N = 28) & Obese (N = 32) & $\boldsymbol{P}_{\text {value* }}^{*}$ \\
\hline CRP & $1(0-1)$ & $2(1-4)$ & 0.001 \\
FEV ${ }_{1}$ & $60(59-70)$ & $64(55-71)$ & 0.882 \\
Sputum eosinophils & $2(2-3)$ & $6(4-7)$ & 0.001 \\
\hline
\end{tabular}

CRP — C-reactive protein; $\mathrm{FEV}_{1}$ — forced expiratory volume in one second

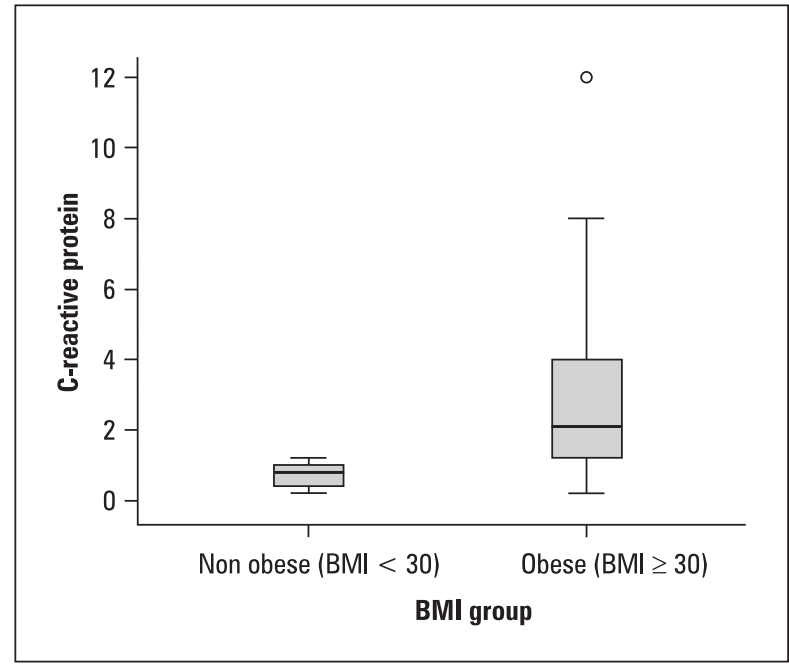

Figure 1. Comparison of C-reactive protein between obese and nonobese asthmatics. BMI — body mass index

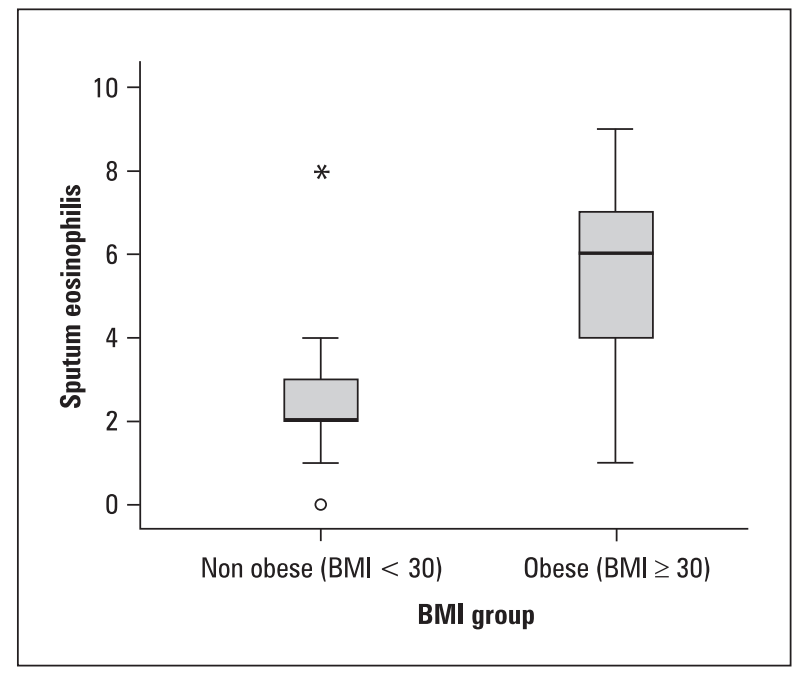

Figure 2. Comparison of sputum eosinophils between obese and nonobese asthmatics 
Table 2. Association between outcomes in obese and nonobese asthmatics

\begin{tabular}{lcccc}
\hline & & Outcome & FEV $_{1}$ & Sputum eosinophils \\
\hline Non-obese $(\mathrm{N}=28)$ & Correlation* & CRP & 0.069 & 0.080 \\
& P value & & 0.358 & 0.337 \\
& Correlation* & FEV & -0.221 & 0.120 \\
& P value & & & 0.301 \\
Obese $(\mathrm{N}=32)$ & Correlation* & CRP & & 0.053 \\
& P value & & -0.037 & -0.054 \\
& Correlation* & FEV & 0.423 & 0.388 \\
All samples $(\mathrm{N}=60)$ & & & 0.520 \\
& P value & CRP & -0.012 & 0.001 \\
& Correlation* & & 0.464 & -0.082 \\
& P value & & & 0.268 \\
\hline
\end{tabular}

*Pearson correlation coefficient. P value $<0.05$ - statistically significant. CRP — C-reactive protein; FEV1 — forced expiratory volume in one second

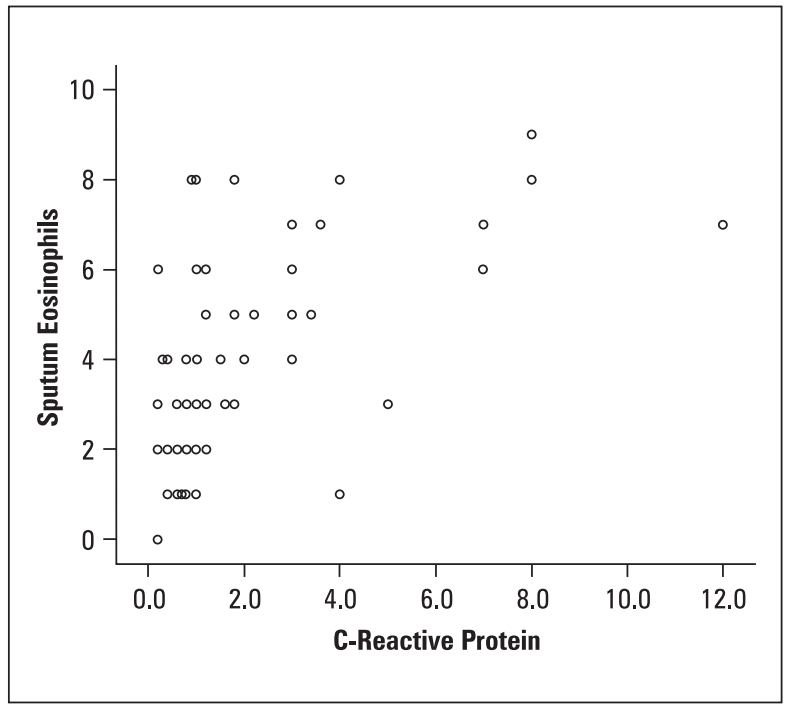

Figure 3. Association between C-reactive protein and sputum eosinophils in all asthmatics

\section{Discussion}

This study aimed to compare the CRP, $\mathrm{FEV}_{1}$ and sputum eosinophils in obese and nonobese asthmatics and to ascertain whether there is an association between the groups. We also sought to find a link between the inflammatory markers - CRP and sputum eosinophils and $\mathrm{FEV}_{1}$, and also among each other in asthmatics and correlate it with asthma control test scores. To the best of our knowledge, this study is one of the very few studies done to compare the various inflammatory markers in both obese and nonobese asthmatics and probably the first study to try to find an association between the inflammatory markers themselves and with the BMI. We also tried to correlate the inflammatory markers with the self-perception of symptom control through the asthma control test.

With our data, we found that median CRP and sputum eosinophils were higher in obese asthmatics than in nonobese asthmatics. Also, CRP and sputum eosinophils had a positive association with BMI. However, the $\mathrm{FEV}_{1}$ did not correlate positively with BMI. Our findings were in concordance with the findings of Van Veen et al. regarding $\mathrm{FEV}_{1}$ that has not decreased in obese asthmatics and has not shown a significant difference between obese and nonobese asthmatics [15]. Interestingly, our study was in contrast to the other finding reported in the same study wherein the authors showed that obese asthmatics do not have more airway inflammation as compared with nonobese asthmatics. But in our study, sputum eosinophils and CRP were also found to be higher in obese asthmatics and had a positive correlation with each other for all asthmatics.

Obese asthmatics had a significant association with asthma control test scores, predominant number of obese asthmatics (94\%) had uncontrolled ACT (score $\leq 19$ ). Even if the $\mathrm{FEV}_{1}$ was independent of the BMI levels, our patients who were obese had an increased perception of symptoms reflected through the poor asthma control scores of less than equal to 19. An increase in systemic inflammation in obesity has been well described [16]. It has been suggested that high levels of pro-inflammatory molecules released from adipose tissue into the systemic circulation could 
Table 3. Association between body mass index (BMI) and asthma control test (ACT)

\begin{tabular}{lccc}
\hline & Non-obese (BMI < 30) & Obese (BMI $\geq \mathbf{3 0})$ & P-value* $^{*}$ \\
\hline Asthma not controlled (ACT $\leq$ 19) & $7(25)$ & $30(94)$ & $\mathbf{0 . 0 0 1}$ \\
Asthma controlled (ACT $>$ 19) & $21(75)$ & $2(6)$ & \\
\hline
\end{tabular}

Numbers with percentage in parenthesis. *Fisher's exact test; $P$ value $<0.05-$ statistically significant

contribute to the airway inflammation, thus increasing the prevalence and poor asthma control in obese asthmatics [17]. In our study, CRP levels were found to be elevated in obese asthmatics and this probably explains that this pro-inflammatory state could lead to increased perception of symptoms. The inflammatory markers (CRP and sputum eosinophils) were independent of the $\mathrm{FEV}_{1}$ in all asthmatics, and thus perception of breathlessness reflected by the poor asthma control test scores $($ ACT $\leq 19)$ in these obese phenotypes of asthmatics could indeed be caused by the systemic inflammation. Many other studies have also demonstrated a positive correlation between elevated CRP levels and asthma control, respiratory impairment and bronchial hyper-reactivity [18-21].

Among the associations between the inflammatory mediators themselves, the CRP and sputum eosinophils showed a positive correlation in all asthmatics. Our study was in agreement with a similar study by Abdelsadek et al. who showed a positive correlation with CRP and sputum eosinophils in asthmatics [22]. In this study, however, the BMI wasn't correlated with the inflammatory markers. The mechanism of airway inflammation is complex in obese asthmatics. It can be mediated through various inflammatory mediators like interleukin (IL)-4, IL-5, IL-13, inflammatory cells like eosinophils, mast cells and basophils to name a few apart from the possible overlay of systemic inflammation. Non-eosinophilic inflammation and systemic inflammation could also play an important role in airway inflammation in obese asthmatics.

Obesity has been demonstrated to be a risk factor for asthma and is associated with an increased prevalence of asthma symptoms [23, $24]$. But this, whether it is because of systemic inflammation, airway constriction or the change in dynamics of respiration and the restrictive defect or a contributory factor of both, needs to be investigated.

As the asthma treatment guidelines are evolving from a symptom-based approach to a tailored approach, it will be wise to optimise the treatment based on various phenotypes and endotypes, and thus probably reducing an impending exacerbation. Interestingly, many studies have previously shown that treating the airway inflammation led to better asthma control and thus, in turn, reduced hospitalisations and fatal events [25], and if the treatment strategy is aimed at keeping sputum eosinophils low, patients might have fewer asthma exacerbations [26]. Since elevated CRP is often associated with accelerated lung function decline [27], aiming at the treatment based on markers of inflammation both the airway and systemic inflammation is a more scientific and rational approach than treating the physiological effects caused by it, which is particularly relevant, especially in obese asthmatics. These obese asthmatics who have an overlay of systemic inflammation could also be phenotyped as a separate entity. In our study, the CRP and sputum eosinophils were higher in obese asthmatics, which suggests concordance between these biomarkers; and similarly to treating the airway inflammation, whether treating the systemic inflammation in these obese phenotypes leads to better asthma control, needs to be examined. Thus, the measure of systemic inflammatory markers in obese asthmatics with poor disease and symptom control plays an important role, and this should help to ascertain the systemic mediator's role and thus help to devise a treatment plan for these subsets of patients. This should ideally include rigorous weight management plans apart from pharmacological interventions in obese asthmatics which aim at reducing the systemic inflammatory mediators like the CRP levels. This might yield a better asthma control wherein the contributors of breathlessness can also be caused by restrictive lung defect in obesity. Apart from their airway inflammation, measuring the systemic inflammation adds a definitive value in difficult to treat obese asthmatics with poor symptom control. It is hoped that these results will help to understand the systemic inflammation of the obese phenotype of asthma and provide better asthma management. Serial measurements of CRP, sputum eosinophils and $\mathrm{FEV}_{1}$, additional interventions and follow-ups 
could have given us more insights into these parameters in different disease states and during various levels of asthma controls.

\section{Conclusions}

Thus, the inflammatory markers sputum eosinophils and CRP were raised in obese asthmatics and had a positive association with BMI. Obese asthmatics had a poorer subjective asthma control than the nonobese asthmatics despite $\mathrm{FEV}_{1}$ being independent of the BMI levels. Measuring the systemic inflammatory markers in obese asthmatics who do not have adequate subjective symptom control reflected by uncontrolled ACT scores, could add a definitive value and possibly help in additional interventions aimed at reducing systemic inflammation and facilitating better symptom control.

\section{Acknowledgments}

We thank the biostatistician Mr. Sriram Ramachandran and all the patients who participated in this study.

\section{Conflict of interest}

None declared.

\section{References:}

1. Kay AB. The role of eosinophils in the pathogenesis of asthma. Trends Mol Med. 2005; 11(4): 148-152, doi: 10.1016/i. molmed.2005.02.002, indexed in Pubmed: 15823751.

2. Corrigan CJ, Haczku A, Gemou-Engesaeth V, et al. CD4 T-lymphocyte activation in asthma is accompanied by increased serum concentrations of interleukin-5. Effect of glucocorticoid therapy. Am Rev Respir Dis. 1993; 147(3): 540-547, doi: 10.1164/ajrccm/147.3.540, indexed in Pubmed: 8095124.

3. Patel DN, King CA, Bailey SR, et al. Interleukin-17 stimulates C-reactive protein expression in hepatocytes and smooth muscle cells via p38 MAPK and ERK1/2-dependent NFkappaB and C/EBPbeta activation. J Biol Chem. 2007; 282(37): 27229-27238, doi: 10.1074/jbc.M703250200, indexed in Pubmed: 17652082 .

4. Pradhan AD, Manson JE, Rifai N, et al. C-reactive protein, interleukin 6 , and risk of developing type 2 diabetes mellitus. JAMA. 2001; 286(3): 327-334, doi: 10.1001/jama.286.3.327, indexed in Pubmed: 11466099.

5. Roberts WL, Moulton L, Law TC, et al. Evaluation of nine automated high-sensitivity C-reactive protein methods: implications for clinical and epidemiological applications. Part 2. Clin Chem. 2001; 47(3): 418-425, indexed in Pubmed: 11238291.

6. Varraso R, Siroux V, Maccario J, et al. Asthma severity is associated with body mass index and early menarche in women. Am J Respir Crit Care Med. 2005; 171(4): 334-339, doi: 10.1164/rccm.200405-674OC, indexed in Pubmed: 15557134.

7. Saint-Pierre P, Bourdin A, Chanez P, et al. Are overweight asthmatics more difficult to control? Allergy. 2006; 61(1): 79-84, doi: 10.1111/j.1398-9995.2005.00953.x, indexed in Pubmed: 16364160.

8. The ENFUMOSA cross-sectional European multicentre study of the clinical phenotype of chronic severe asthma.
European Network for Understanding Mechanisms of Severe Asthma. Eur Respir J. 2003; 22(3): 470-477, doi: 10.1183/09031936.03.00261903, indexed in Pubmed: 14516137.

9. Ford ES. The epidemiology of obesity and asthma. J Allergy Clin Immunol. 2005; 115(5): 897-909; quiz 910, doi: 10.1016/j. jaci.2004.11.050, indexed in Pubmed: 15867841.

10. Desai D, Newby C, Symon FA, et al. Elevated sputum interleukin-5 and submucosal eosinophilia in obese individuals with severe asthma. Am J Respir Crit Care Med. 2013; 188(6): 657-663, doi: 10.1164/rccm.201208-1470OC, indexed in Pubmed: 23590263.

11. Scott HA, Gibson PG, Garg ML, et al. Airway inflammation is augmented by obesity and fatty acids in asthma. Eur Respir J. 2011; 38(3): 594-602, doi: 10.1183/09031936.00139810, indexed in Pubmed: 21310876.

12. Thomas M, Kay S, Pike J, et al. The Asthma Control Test (ACT) as a predictor of GINA guideline-defined asthma control: analysis of a multinational cross-sectional survey. Prim Care Respir J. 2009; 18(1): 41-49, doi: 10.4104/pcrj.2009.00010, indexed in Pubmed: 19240948.

13. Bartlett J, Breiman R, Mandell L, et al. Community Acquired pneumonia in adults: guidelines for management. Clinical Infectious Diseases. 1998; 26(4): 811-838, doi: 10.1086/513953.

14. Pavord ID, Brightling CE, Woltmann G, et al. Non-eosinophilic corticosteroid unresponsive asthma. Lancet. 1999; 353(9171): 2213-2214, doi: 10.1016/S0140-6736(99)01813-9, indexed in Pubmed: 10392993.

15. van Veen IH, Ten Brinke A, Sterk PJ, et al. Airway inflammation in obese and nonobese patients with difficult-to-treat asthma. Allergy. 2008; 63(5): 570-574, doi: 10.1111/j.13989995.2007.01597.x, indexed in Pubmed: 18394131.

16. Lessard A, Turcotte H, Cormier Y, et al. Obesity and asthma: a specific phenotype? Chest. 2008; 134(2): 317-323, doi: 10.1378/chest.07-2959, indexed in Pubmed: 18641097.

17. Shore SA, Johnston RA. Obesity and asthma. Pharmacol Ther. 2006; 110(1): 83-102, doi: 10.1016/j.pharmthera.2005.10.002, indexed in Pubmed: $\underline{16297979}$.

18. Szalai AJ. C-reactive protein (CRP) and autoimmune disease: facts and conjectures. Clin Dev Immunol. 2004; 11(3-4): 221226, doi: 10.1080/17402520400001751, indexed in Pubmed: 15559367.

19. Jousilahti P, Salomaa V, Hakala K, et al. The association of sensitive systemic inflammation markers with bronchial asthma. Annals of Allergy, Asthma \& Immunology. 2002; 89(4): 381-385, doi: 10.1016/s1081-1206(10)62039-x.

20. Ford ES. Asthma, body mass index, and C-reactive protein among US adults. J Asthma. 2003; 40(7): 733-739, doi: 10.1081/jas-120023497, indexed in Pubmed: 14626329.

21. Gan WQ, Man SFP, Senthilselvan A, et al. Association between chronic obstructive pulmonary disease and systemic inflammation: a systematic review and a meta-analysis. Thorax. 2004; 59(7): 574-580, doi: 10.1136/thx.2003.019588, indexed in Pubmed: 15223864.

22. Al-Aarag Abdelsadek H, Rawy Abeer M, EL-Behissy Mona M, et al. Study of serum C-reactive protein level and sputum eosinophils in patients with bronchial asthma. E J bronchology. 2015; 9(1): 43-47, doi: 10.4103/1687-8426.153597.

23. Beuther DA, Sutherland ER. Overweight, obesity, and incident asthma: a meta-analysis of prospective epidemiologic studies. Am J Respir Crit Care Med. 2007; 175(7): 661-666, doi: 10.1164/ rccm.200611-17170C, indexed in Pubmed: 17234901.

24. Shore SA. Obesity and asthma: implications for treatment. Curr Opin PulmMed. 2007; 13: 5.

25. Miranda C, Busacker A, Balzar S, et al. Distinguishing severe asthma phenotypes: role of age at onset and eosinophilic inflammation. J Allergy Clin Immunol. 2004; 113(1): 101-108, doi:10.1016/j.jaci.2003.10.041, indexed in Pubmed: 14713914.

26. Siva R, Green RH, Brightling CE, et al. Asthma exacerbations and sputum eosinophil counts: a randomised controlled trial. Lancet. 2002; 360(9347): 1715-1721, doi: 10.1016/S01406736(02)11679-5, indexed in Pubmed: 12480423.

27. Rasmussen F, Mikkelsen D, Hancox RJ, et al. High-sensitive C-reactive protein is associated with reduced lung function in young adults. Eur Respir J. 2009; 33(2): 382-388, doi: 10.1183/09031936.00040708, indexed in Pubmed: 19010993. 